\title{
Novo coronavírus: sentimentos vivenciados por profissionais de um hospital público que realizaram exame de detecção viral
}

New coronavirus: feelings experienced by professionals from a public who underwent viral

\author{
detection exam
}

Nuevo coronavirus: sentimientos experimentados por profesionales de un hospital que realizaron un examen de detección viral

Kelly Elaine de Sousa ORCID: https://orcid.org/0000-0001-7936-9561 Universidade Estadual de Maringá, Brasil E-mail: sousakelly1@gmail.com

Rafaela Ferreira de Oliveira ORCID: https://orcid.org/0000-0002-3651-5351 Universidade Estadual de Maringá, Brasil E-mail: rafafff@outlook.com

Rosimara Oliveira Queiroz ORCID: https://orcid.org/0000-0001-7976-2259 Universidade Estadual de Maringá, Brasil E-mail: rosi.mdc@hotmail.com Marcelo da Silva ORCID: https://orcid.org/0000-0002-0376-0430 Universidade Estadual de Maringá, Brasil E-mail: marceloascencio@gmail.com

Sonia Silva Marcon

ORCID: https://orcid.org/0000-0002-6607-362X Universidade Estadual de Maringá, Brasil E-mail: soniasilva.marcon@gmail.com

Herbert Leopoldo de Freitas Goes ORCID: https://orcid.org/0000-0002-6071-692X Universidade Estadual de Maringá, Brasil E-mail: hlfgoes@gmail.com

\begin{abstract}
Resumo
Objetivo: apresentar os sentimentos e perspectivas dos profissionais de saúde em relação à detecção do novo coronavírus. Metodologia: estudo descritivo-exploratório de natureza qualitativa, desenvolvido junto a 73 profissionais de um hospital do noroeste do Paraná. Os dados foram coletados através de entrevistas semiestruturadas, processados na Interface de R pour les Analyses Multidimensionnelles de Textes et de Questionnaires (IRAMUTEQ) utilizando-se do recurso de nuvem de palavras. Resultados: a análise das entrevistas possibilitou a construção de três categorias temática: Motivação para a realização do teste rápido para Coronavírus; Protocolos utilizados para a realização do teste rápido nos profissionais e Sentimentos vivenciados pelos servidores frente à possibilidade de confirmação de um caso positivo na equipe. Conclusão: diante da percepção dos profissionais envolvidos nesta pesquisa, pode se confirmar que enfrentamento da Covid-19, representa um estressor demostrando que a preocupação e medo no contexto da pandemia, muitas vezes estavam associados aos sentimentos dos participantes em adquirir o vírus e transmitir para sua família ou pessoas que amam.
\end{abstract}

Palavras-chave: Coronavírus; SARS-CoV-2; Covid-19; Profissionais da saúde.

\section{Abstract}

Objective: present the feelings and perspectives of health professionals regarding the detection of the new coronavirus Methodology: descriptive-exploratory study of a qualitative nature, developed with 73 professionals from a hospital in the northwest of Paraná. The data were collected through semi-structured interviews, processed in the Interface of $\mathrm{R}$ pour les Analyzes Multidimensionally de Texts et de Questionnaires (IRAMUTEQ) using the word cloud resource. Results: the analysis of the interviews allowed the construction of three thematic categories: Motivation to perform the rapid test for Coronavirus; Protocols used to perform the rapid test in the professionals and Feelings experienced by the employees regarding the possibility of confirming a positive case in the team. Conclusion: given the perception of the professionals involved in this research, it can be confirmed that coping with Covid-19 represents a stressor 
showing that the concern and fear in the context of the pandemic, were often associated with the feelings of the participants in acquiring the virus and transmitting it to your family or people you love.

Keywords: Coronavírus; SARS-CoV-2; Covid-19; Professional of health.

\begin{abstract}
Resumen
Objetivo: presentar los sentimientos y perspectivas de los profesionales sobre la posibilidad frente a la detección del nuevo coronavirus. Analice los factores que influyen en la detección del covid-19. Metodología: estudio cualitativo descriptivo-exploratorio, desarrollado con 73 profesionales de un hospital del noroeste de Paraná. Los datos fueron recolectados a través de entrevistas semiestructuradas, procesadas en el Interface de $R$ pour les Analyses Multidimensionnelles de Textes et de Questionnaires (IRAMUTEQ) utilizando el recurso de nube de palabras. Resultados: el análisis de las entrevistas permitió la construcción de tres categorías temáticas: Motivación para realizar la prueba rápida para Coronavirus; Protocolos utilizados para realizar la prueba rápida en los profesionales y Sentimientos experimentados por los empleados sobre la posibilidad de confirmar un caso positivo en el equipo. Conclusión: dada la percepción de los profesionales involucrados en esta investigación, se puede confirmar que el afrontamiento del Covid-19 representa un estresor, mostrando que la preocupación y el miedo en el contexto de la pandemia, a menudo se asociaron con los sentimientos de los participantes en adquirir el virus y transmitirlo a su família os personas que ama.
\end{abstract}

Palabras clave: Coronavirus; SARS-CoV-2; COVID-19; Profesionales de la salud.

\title{
1. Introdução
}

O SARS-CoV-2, o novo coronavírus responsável pela pandemia atual de Covid-19 surgiu como um problema global em poucos meses após sua primeira descrição em Wuhan-China. Além de sua taxa de mortalidade alarmante e de natureza facilmente transmissível por meio de gotículas de ar, tornou-se uma séria ameaça à saúde pública em todo o mundo, com milhões de pessoas em risco (Harapan, et al., 2019).

Os novo coronavírus são um grupo de vírus conhecidos por causar doenças que podem ir de uma simples gripe a uma pneumonia atípica, sendo conhecidos até o momento 7 tipos de coronavírus, incluindo o SARS-CoV-2, que causa o COVID-19 (WHO, 2011).

Sabe-se que a sua forma de transmissibilidade provoca uma síndrome respiratória aguda, evidências indicam que a máscara facial ajuda a prevenir a transmissão do novo coronavírus humanos, autoridades de saúde têm recomendado o uso de máscara facial em esferas públicas para autoproteção. Sua letalidade se modifica, principalmente, conforme a faixa etária e condições clínicas associadas (Brasil, 2001).

Estudos estatísticos revelam que, em média, um paciente infectado pode levar a 5/7 novos casos confirmados subsequentes. Então, desde o primeiro caso da doença do Coronavírus em 2019, houve um total de 60.412 casos confirmados com 1370 fatalidades relatadas em 25 países diferentes, surgindo graves impactos na saúde social, mental e na economia dos países afetados. (Yan, et al., 2020).

A pandemia do novo Coronavírus provocou um clima generalizado de cautela e incerteza, principalmente entre os profissionais de saúde, devido à facilidade de disseminação, e gravidade dos sintomas, desconhecimento da doença e óbitos entre profissionais de saúde (Choi, 2019). Existe um consenso em toda a literatura de que os profissionais de saúde correm risco de implicações psicológicas principalmente por demonstrarem preocupação com a transmissão para sua família, especialmente envolvendo os idosos, imunocomprometidos ou com condições médicas crônicas (Adams \& Rom, 2020; Neves, et al., 2020).

Diante da pandemia os profissionais tiveram que se adaptar rapidamente com o medo do contagio e transmissão do vírus, trazendo emoções de angustia que podem enfraquecer a confiança desses profissionais em sua capacidade de permanecer calmo e tranquilizar o público (Adams \& Rom, 2020). A transmissão nosocomial é conhecida como uma infecção adquirida no ambiente hospitalar podendo se disseminar entre profissionais da saúde que atuam na linha de frente ao combate da doença e têm sido motivo de discussão entre os profissionais e gestores, evidenciando a necessidade de estudos e avaliação dos riscos no ambiente de hospital. 
Preocupações com a saúde mental, ajuste psicológico dos profissionais de saúde que tratam e cuidam de pacientes com o novo coronavírus, estão surgindo, nesse contexto é necessário realizar intervenções para promover o bem-estar mental, além de fortalecer estratégias de prevenção e gerenciamento de crises entre os trabalhadores de saúde (Shanafelt, et al., 2020).

Desta forma tolera-se em buscar, evidências a respeito do seguinte questionamento: Quais os sentimentos vivenciados pelos profissionais que estão na linha de frente, diante possibilidade de confirmação de casos positivo na equipe?

Nesse sentido, objetivo do estudo é apresentar os sentimentos e perspectivas dos profissionais de saúde em relação à detecção do novo coronavírus.

\section{Metodologia}

Estudo descritivo-exploratório de natureza qualitativa, para compreender melhor os sentimentos vivenciados pelos profissionais frente à possibilidade de confirmação de um caso positivo na equipe. Este referencial está voltado para as experiências dos sujeitos dentro do ambiente de trabalho, que aparecem pela relação sofrimento-prazer e pelo incremento de táticas que permitem um ambiente seguro (Mendes, 2007).

O presente estudo foi realizado em um hospital municipal de médio porte, da Região Noroeste do Paraná. O hospital atualmente é composto por pronto socorro com uma sala de emergência, sala de curativos, sala de sutura, sala de injeção, seis consultórios médicos, clínica médica de internamento, com 22 leitos, sendo um para isolamento, pediatria com cinco leitos. O hospital possui uma área exclusiva para o atendimento de pacientes suspeitos ou positivos para o novo coronavírus, com uma sala de emergência, sala de medicação, um consultório médico e 12 leitos de internação.

Os sujeitos da pesquisa foram todos os profissionais que trabalham na referida instituição, sendo que atualmente o hospital possui cerca de 120 servidores, composta por médicos, enfermeiros, auxiliares e técnicos de enfermagem, farmacêuticas, auxiliares de farmácia, nutricionista, equipe administrativa, coordenação e serviços gerais.

Como critérios de exclusão do estudo, foram todos os servidores do hospital, que por sua vez estavam de férias, folga ou licença saúde.

O quantitativo de profissionais abordados foi definido pelos critérios de saturação teórica, isto é, quando as informações coletadas se tornaram repetitivas e permitiram responder aos objetivos do estudo (Fontanella, et al., 2008).

A coleta de dados foi realizada mediante entrevistas individuais, agendados em local e horário de interesse do entrevistado, em sala reservada no período matutino, vespertino e noturno, as quais foram guiadas por um instrumento semiestruturado constituído por duas partes: uma abordando características sociodemográficas e outra com questões abertas acerca dos sentimentos e perspectivas dos profissionais frente à possibilidade de confirmação de um caso positivo na equipe.

O instrumento de coleta de dados foi enviado a três juízes, que procederam à análise das questões. Após a aprovação das questões pelos juízes, foi realizado o convite por meio de contato pessoal e via telefone. Mediante o aceite verbal foi realizada as entrevistas em local e horário definido pelo participante. As entrevistas foram registradas em um gravador mp3. Após a gravação os depoimentos foram transcritos na íntegra, estruturados em categorias e analisados qualitativamente por meio da Análise de Conteúdo de Bardin (Bardin, 2011).

A interpretação dos resultados e a discussão discorre sobre as relações entre trabalho e saúde, principalmente sobre os aspectos psicológicos da saúde mental, na qual o trabalho pode exercer influência, e as estratégias defensivas adotadas pelos trabalhadores em situações de estresse.

Em seguida foram analisados os depoimentos por lexicografia básica utilizando o software Interface de R por les Analyses Multidimensionnelles de Texte set de Questionnaires (IRaMuTeQ®). Por meio dele, foi utilizada a Nuvem de Palavras, que organiza de modo gráfico as palavras em função da frequência que aparecem nos discursos (Marchant \& Ratinald, 2012). 
O estudo seguiu os preceitos éticos vigentes na Resolução n 466/2012 do Conselho Nacional de Saúde e seu projeto foram aprovados pelo Comitê de Ética em Pesquisa da Universidade Estadual de Maringá, sob o número do parecer 4.107.276 (Brasil, 2012). Todos os participantes assinaram o Termo de Consentimento Livre e Esclarecido em duas vias. Para preservar o anonimato, os trechos dos relatos estão identificados pelas iniciais da sua profissão, seguido por numeral arábico conforme a ordem das entrevistas, a fim de garantir o anonimato das respostas.

\section{Resultados}

\section{Caracterização dos Participantes}

Foram entrevistados 73 servidores, de um hospital municipal localizado na Região Noroeste do Paraná. Esses profissionais, em sua grande maioria não apresentam comorbidades (75,3\%), estão na faixa etária de 40 a 50 anos (60,3\%), sendo do sexo feminino $(69,9 \%)$, apresentam ter de 1 a 2 filhos $(64,4 \%)$, casados $(61,6 \%)$, com formação de ensino técnico $(49,3 \%)$ e sem alguma especialização (72,6\%), conforme Tabela 1.

Tabela 1 - Perfil dos servidores de um hospital em atendimento ao Coronavírus - região Noroeste do Paraná em 2020.

\begin{tabular}{|c|c|c|c|c|c|c|c|c|c|c|c|c|c|c|}
\hline \multirow{3}{*}{ Características } & \multicolumn{10}{|c|}{ Categoria Profissional } & & & & \\
\hline & \multicolumn{2}{|c|}{ ENF* } & \multicolumn{2}{|c|}{ Medicina } & \multicolumn{2}{|c|}{$\begin{array}{c}\text { Tec. de } \\
\text { Radiologia }\end{array}$} & \multicolumn{2}{|c|}{ Farmácia } & \multicolumn{2}{|c|}{ ADM* } & \multicolumn{2}{|c|}{$\begin{array}{c}\text { Serviços } \\
\text { Gerais }\end{array}$} & \multicolumn{2}{|c|}{ Total } \\
\hline & $\mathbf{N}$ & $\%$ & $\mathbf{N}$ & $\%$ & $\mathbf{N}$ & $\%$ & $\mathbf{N}$ & $\%$ & $\mathbf{N}$ & $\%$ & $\mathbf{N}$ & $\%$ & $\mathbf{N}$ & $\%$ \\
\hline \multicolumn{15}{|l|}{ Comorbidades } \\
\hline Sim & 7 & 25,0 & - & - & - & - & 1 & 33,3 & 2 & 33,3 & 8 & 40,0 & 18 & 24,7 \\
\hline Não & 21 & 75,0 & 11 & 100,0 & 5 & 100,0 & 2 & 66,7 & 4 & 66,7 & 12 & 60,0 & 55 & 75,3 \\
\hline \multicolumn{15}{|l|}{$N^{0}$ de filhos } \\
\hline Nenhum & 5 & 17,9 & 5 & 45,5 & 1 & 20,0 & 1 & 33,3 & 4 & 66,7 & 1 & 5,0 & 17 & 23,3 \\
\hline 1 a 2 filhos & 22 & 78,6 & 5 & 45,5 & 4 & 80,0 & 1 & 33,3 & 2 & 33,3 & 13 & 65,0 & 47 & 64,4 \\
\hline 3 a 4 filhos & 1 & 3,6 & 1 & 9,0 & - & - & 1 & 33,3 & - & - & 5 & 25,0 & 8 & 11,0 \\
\hline 5 a 6 filhos & - & - & - & - & - & - & - & - & - & - & 1 & 5,0 & 1 & 1,4 \\
\hline \multicolumn{15}{|l|}{ Sexo } \\
\hline Feminino & 23 & 82,1 & 5 & 45,5 & 1 & 20,0 & 3 & 100,0 & 4 & 66,7 & 15 & 75,0 & 51 & 69,9 \\
\hline Masculino & 5 & 17,9 & 6 & 54,5 & 4 & 80,0 & - & - & 2 & 33,3 & 5 & 25,0 & 22 & 30,1 \\
\hline \multicolumn{15}{|l|}{ Idade } \\
\hline 20-30 anos & 11 & 39,3 & 6 & 54,5 & 2 & 40,0 & 1 & 33,3 & 3 & 50,0 & 6 & 30,0 & 29 & 39,7 \\
\hline $40-50$ anos & 17 & 60,7 & 5 & 45,5 & 3 & 60,0 & 2 & 66,7 & 3 & 50,0 & 14 & 70,0 & 44 & 60,3 \\
\hline \multicolumn{15}{|l|}{ Estado Civil } \\
\hline Solteiro & 5 & 17,9 & 2 & 18,2 & 1 & 20,0 & - & - & 3 & 50,0 & 4 & 20,0 & 15 & 20,5 \\
\hline Casado & 19 & 67,9 & 7 & 63,6 & 3 & 60,0 & 3 & 100,0 & 3 & 50,0 & 10 & 50,0 & 45 & 61,6 \\
\hline Divorciado & 4 & 14,3 & 2 & 18,2 & 1 & 20,0 & - & - & - & - & 6 & 30,0 & 13 & 17,8 \\
\hline \multicolumn{15}{|l|}{$\begin{array}{l}\text { Nível de } \\
\text { Formacão }\end{array}$} \\
\hline $\begin{array}{l}\text { Ensino } \\
\text { Fundamental }\end{array}$ & - & - & - & - & - & - & - & - & - & - & 3 & 15,0 & 3 & 4,1 \\
\hline Ensino Médio & - & - & - & - & - & - & - & - & - & - & 5 & 25,0 & 5 & 6,8 \\
\hline Técnico & 16 & 57,1 & - & - & 5 & 100,0 & 3 & 100,0 & - & - & 12 & 60,0 & 36 & 49,3 \\
\hline Superior & 12 & 42,9 & 11 & 100,0 & - & - & - & - & 3 & 50,0 & - & - & 26 & 35,6 \\
\hline \multicolumn{15}{|l|}{ Especialização } \\
\hline Sim & 11 & 39,3 & 4 & 36,4 & - & - & 1 & 33,3 & 4 & 66,7 & - & - & 20 & 27,4 \\
\hline Não & 17 & 60,7 & 7 & 63,6 & 5 & 100,0 & 2 & 66,7 & 2 & 33,3 & 20 & 100,0 & 53 & 72,6 \\
\hline
\end{tabular}

Legenda: *ENF= Enfermeiros, Técnicos e Auxiliares de Enfermagem; ADM= Administrativo. Fonte: Autores (2020). 
Evidencia-se que os servidores desse hospital apresentam de 1 a 4 anos de trabalho (37\%), com carga horária de 30 a 36 horas semanal (52,1\%), trabalhando no período diurno (72,6\%), com um vínculo empregatício (74\%), conforme Tabela 2.

Tabela 2 - Características Trabalhistas dos Profissionais do Hospital.

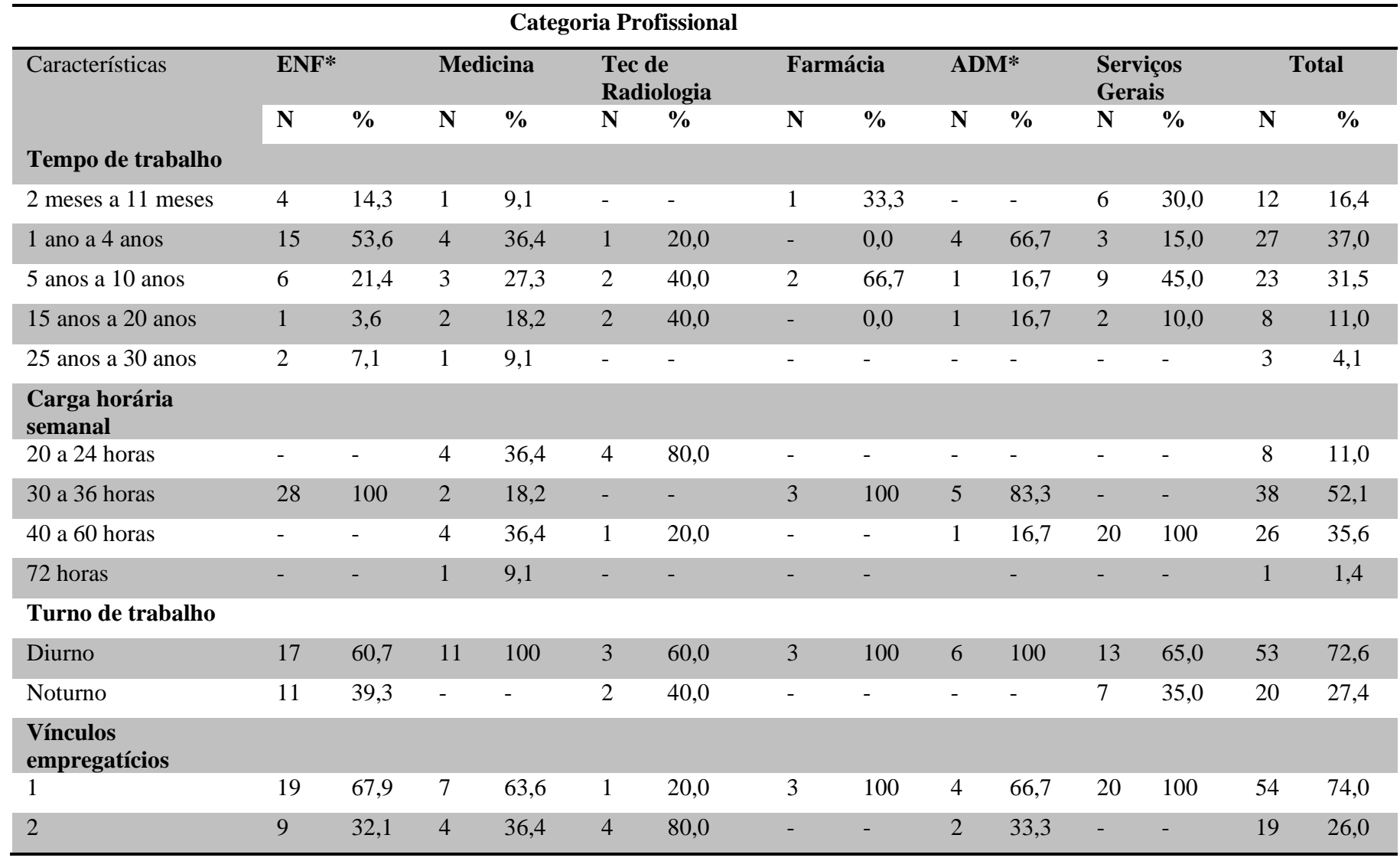

Legenda: *ENF= Enfermeiros, Técnicos e Auxiliares de Enfermagem; ADM= Administrativo. Fonte: Autores (2020).

A análise das entrevistas possibilitou a construção de três categorias temáticas: (1) Motivação para a realização do teste rápido para coronavírus; (2) Protocolos utilizados para a realização do teste rápido nos profissionais; (3) Expectativas vivenciadas pelos trabalhadores frente à possibilidade de confirmação de um caso positivo na equipe.

\section{Motivação para a realização do teste rápido para Covid-19}

Sobre as Motivações para realização do teste rápido para Coronavírus, verificou-se que a palavra (Positivo) apresentou maior frequência no corpus um $(n=23)$, seguido da palavra (Covid) $(n=22)$, contato ( $n=20)$, vírus ( $n=11)$, instituição $(n=11)$, conforme Figura 1. 
Figura 1 - Nuvem de Palavra para eixo Motivação para a Realização do Teste Rápido para Coronavírus.

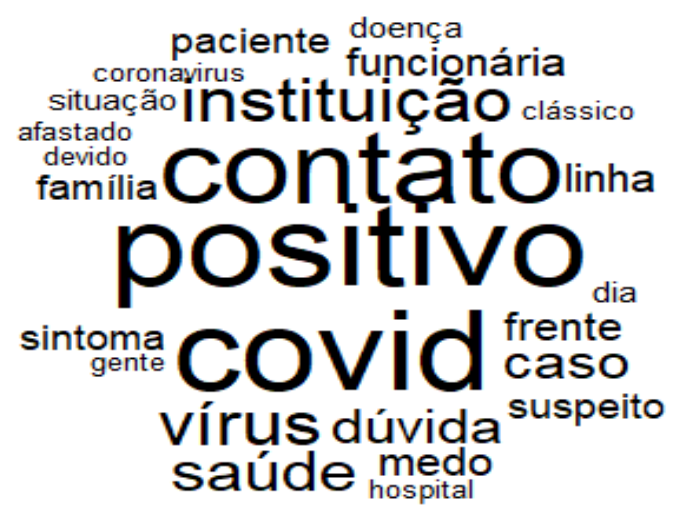

Fonte: Autores (2020).

Pode-se verificar que a grande maioria dos profissionais apresentavam interesse em realizar o teste rápido pelo fato de estarem atuando diariamente com pacientes suspeitos e positivos para o vírus, e em outras situações, encontram-se também os profissionais que não atuam diretamente com pacientes como, por exemplo, os profissionais administrativos.

Sim, pois trabalhamos dentro de um hospital que recebe diariamente casos suspeitos e positivos para Covid 19 e estamos constantemente entrando em ambiente que atendem essas pessoas. (S9)

Porque a gente trabalha na linha de frente do atendimento aos pacientes suspeitos ou confirmados para Covid-19. (E12).

Sim, pois estou na linha de frente do Covid 19 e consequentemente acabo lidando com pacientes suspeitos ou confirmados para Covid 19. (C4)

Protocolos utilizados para a realização do teste rápido nos profissionais

Sobre o questionamento quem deveriam fazer o teste rápido para Covid-19, verificou-se que a palavra contato apresentou maior frequência $(n=32)$, seguido da palavra covid $(n=31)$, positivo $(n=25)$ e todos os profissionais da saúde $(\mathrm{n}=14)$, conforme Figura 2.

Figura 2 - Nuvem de Palavras para eixo Protocolos Utilizada para a Realização do Teste Rápido nos Profissionais.

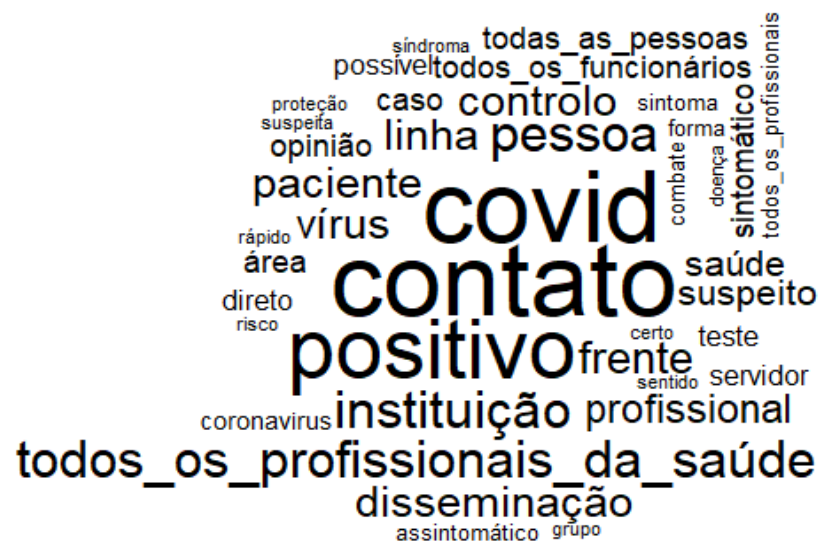

Fonte: Autores (2020). 
Destacou-se que as pessoas que deveriam realizar o teste para Covid-19 se tratava de profissionais de saúde, que diariamente estão operando nos cuidados voltados aos pacientes suspeitos ou positivos para o novo Coronavírus.

Todos os profissionais da saúde que trabalham na linha de frente do Covid 19, pois diariamente estão em contato direto com paciente suspeito positivo para o coronavírus. (S7)

Todos os profissionais da Saúde que estão na linha de frente do covid-19 para o controle da disseminação do vírus dentro do ambiente hospitalar no sentido de minimizar os afastamentos e consequentemente e não prejudicar o ambiente de trabalho. (T12)

Todos os profissionais da saúde que estão na linha de frente do Covid 19 e todos os pacientes com Síndrome gripal. (M4)

\section{Expectativas vivenciadas pelos trabalhadores frente à possibilidade de confirmação de um caso positivo na equipe}

No que se refere às expectativas verificou-se que as palavras mais frequentes foram: Medo ( $\mathrm{n}=26)$ Preocupação (n=18), Vírus (n=12), Família (n=8), conforme Figura 3.

Figura 3 - Nuvem de Palavras para Eixo Expectativas Vivenciadas pelos Profissionais da Saúde Frente à Possibilidade de Confirmação de um Caso Positivo na Equipe.

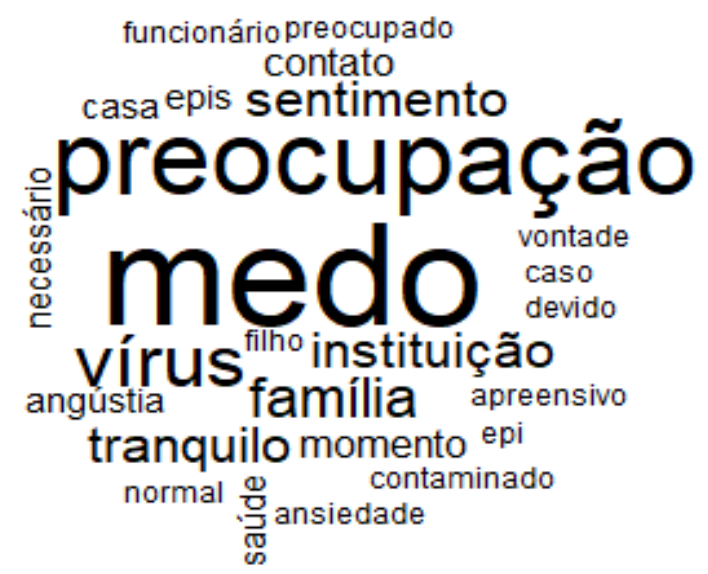

Fonte: Autores (2020).

Evidenciou que as palavras preocupação e medo no contexto da pandemia muitas vezes estavam associados aos sentimentos dos participantes em adquirir o vírus e transmitir para sua família ou pessoas que amam.

Medo e preocupação, pois podemos disseminar no ambiente de trabalho devido aos pacientes internados e por nós, que estamos em contato com a família e pessoas de terceira idade que incluem hipertensos e cardíacos. (E10)

Preocupação com os demais e com a possibilidade de estar disseminando o vírus. (F2)

Medo de estar com vírus e passar para as pessoas que eu amo. (S2)

\section{Discussão}

Os profissionais de saúde são um grupo que possui grande risco para a Covid-19 por estarem expostos diretamente aos pacientes infectados, estão submetidos ao estresse ao atender pacientes em estado crítico, aumentando a exposição de risco ao COVID-19. O aumento dos sintomas de ansiedade, depressão, perda da qualidade do sono, aumento do uso de drogas, sintomas psicossomáticos e medo de se infectarem ou transmitirem a infecção aos membros da família (Fiocruz, 2021). 
A covid-19 está causando de saúde a nível mental, como estresse, ansiedade, emoções negativas, associado a falta de contato com entes próximos, com sintomas de exaustão no ambiente laboral. O medo de ser infectado, a proximidade com o sofrimento dos pacientes ou a morte destes, solidão e preocupações com entes queridos (Huang, et al., 2020).

O enfrentamento da pandemia do novo coronavírus faz parte das funções e responsabilidade dos profissionais da saúde que atuam no processo de saúde-doença em seus diferentes níveis de atenção, com ações de promoção, prevenção, recuperação e reabilitação à saúde, por meio de ações voltadas para a população ou para grupos com maior risco de contaminação, como os profissionais de saúde (Baker, et al., 2020).

Diante da percepção dos profissionais envolvidos nesta pesquisa, pode-se confirmar que muitos profissionais da saúde não têm experiência de atuação em situações de estresse, como é o caso dos atendimentos dos pacientes com Covid-19, o que representa um estressor no ambiente de trabalho.

Com isso, aprender e ensinar, estratégias de enfrentamento, como o autocuidado para que possam dar segurança na assistência prestada, gerenciamento de estresse, e treinamento correto no manejo dos equipamentos de proteção individual (EPI), é imprescindível em momentos de incerteza. Nesse contexto de pandemia, os serviços de saúde necessitam reconhecer e valorizar a percepção dos profissionais atuantes nesse ambiente, a fim de planejar e programar medidas para diminuir o estresse e a insatisfação no trabalho.

Algumas medidas podem ser tomadas como, o desenvolvimento de atividades de educação permanente que valorizem a atuação do profissional e as suas dificuldades cotidianas durante a pandemia, como o manejo adequado dos EPIs, e a tecnologia dura como os respiradores e dispositivos utilizados na assistência direta a pacientes suspeitos ou confirmados do Covid 19. Compete salientar que a formação em técnicas de autocontrole e gestão do estresse reforçam o otimismo e a autoestima do profissional envolvido neste cenário.

Profissionais cuja atividade exige o contato corpo a corpo são mais suscetíveis à contaminação, exemplo são os, enfermeiros, técnicos de enfermagem e médicos que são algumas das categorias que desenvolvem serviços essenciais e estão expostas diariamente ao risco de contaminação de até 15\% a mais de serem infectados pelo vírus SARS-CoV-2 (SESA, 2020). $\mathrm{Na}$ Itália, cerca de $20 \%$ dos profissionais que atuavam no combate ao Covid-19 foram infectados pela doença (Yeh-Li \&, Morais, 2020).

No entanto, não só os profissionais da saúde acabam lidando com a disseminação e o contágio do vírus dentro do ambiente hospitalar. Outros servidores que realizam diferentes atividades nesse meio, também podem contribuir com o aumento da disseminação e contágio, portanto a forma como são realizadas as atividades são decisivas para o adoecimento. Por sua vez, a instituição hospitalar se torna um território de disseminação da doença em qualquer das atividades nela realizadas (Baker, et al., 2020).

As situações, no qual esses profissionais estão expostos criam motivações que estão ligadas a fatores intrínsecos particular de cada pessoa, envolvidas pelo sofrimento causado pelas condições vivenciadas no momento (Costa \& Moura, 2018; Mendes, 2007) Diante dessa situação de constante exposição ao vírus dentro do ambiente de trabalho, os testes rápidos para o diagnóstico do novo coronavírus se mostraram como uma ferramenta primordial na identificação dos casos de infecção, dando segurança aos profissionais a não transmitir o vírus para seus familiares e profissionais (Pan, et al., 2020)

Atualmente existe uma extensa variedade de testes diagnósticos para o Covid-19 disponível no mercado (Costa \& Moura, 2018). Existem os testes que usam sangue, soro ou plasma e os outros que precisam de amostras de secreções coletadas das vias respiratórias, como nasofaringe (nariz) e orofaringe (garganta). Isso proporcionou o mapeamento do status imunológico de uma determinada população, que contribui nas mudanças voltadas para estratégias com o objetivo de controlar a disseminação (Anvisa, 2020). 
Para a realização dos testes rápidos é recomendável que os sujeitos apresentem ou tenham apresentado os sintomas característicos do novo coronavírus há cerca de oito dias (Pan, et al., 2020). Nesse sentido, orienta-se a coleta desde que se enquadrem na definição de síndrome gripal, ou seja, "indivíduo com quadro respiratório agudo, caracterizado por sensação febril ou febre, mesmo que relatada, acompanhada de tosse ou dor de garganta ou coriza ou dificuldade respiratória" (Ministério da saúde, 2020)

A exposição dos profissionais ao vírus, decorrentes do seu trabalho, e em algumas situações a necessidade de se realizar o teste rápido devido às diversas circunstâncias ocorridas dentro do ambiente hospitalar, como apresentados neste estudo, implicaram seriamente na saúde mental de diversos servidores principalmente do que se refere à possibilidade de um caso de confirmação dentro da equipe (Pan, et al., 2018; Ministério da saúde, 2020).

A saúde mental da população diante de uma crise social, causada pelo Covid-19, responsável por problemas voltados para a saúde pública internacional, destaca-se que os efeitos causados pela pandemia acabam tendo maiores repercussões comparadas ao número de mortes. Isso pode significar maior carga de experiências, sentimentos e emoções negativas, provocando a busca de cuidados psicológicos (Brooks, et al; 2020).

Enfermeiros e outros profissionais estão sofrendo estresse físico, estresse emocional e sofrimento moral, por valores profissionais. Esses valores são confrontados com questões éticas e complexas na prática laboral, devido ao medo e a preocupação do adoecimento, da morte e a capacidade de disseminação (Ho, et al., 2020).

O medo está diretamente associado à sua taxa de transmissão rápido e invisível, bem como à sua morbimortalidade. Isso ainda leva a outros desafios psicossociais, incluindo estigmatização, e discriminações. Alto nível de medo faz os indivíduos não pensarem de forma clara e racional ao reagir ao Covid-19. O medo do desconhecido leva a um alto nível de ansiedade, é provável que as respostas emocionais das pessoas incluam comportamentos sociais negativos geralmente conduzidos por percepções de risco (Baker, et al., 2020).

A disseminação do medo e da preocupação influencia não apenas as respostas emocionais às circunstâncias atuais, mas também leva a uma piora dos distúrbios afetivos e de ansiedade, bem como os transtornos obsessivo-compulsivos com seus rituais de limpeza. A saúde mental dos profissionais de saúde da linha de frente contra o Covid-19 não deve ser negligenciada, o impacto de cargas de trabalho extremas, exaustão física e mental, insônia e a ansiedade e o medo de ser infectado ou transmitir infecção a entes é imensurável (Maben \& Bridges, 2020).

Esses profissionais necessitam de coragem para trabalhar na linha de frente da pandemia do Covid-19. Não há dúvida de que os enfermeiros e os outros profissionais continuam a enfrentar os desafios para poder lidar com essas emergências de saúde pública nessa escala. Intervenções especiais para promover o bem-estar mental dos profissionais de saúde expostos ao Covid-19 precisam ser implementadas, portanto apoiar todos os profissionais é essencial para preservar sua saúde a curto e longo prazo, principalmente quando os níveis de estresse ocupacional são tão altos (Yao, et al., 2020; Turale, et al., 2020).

\section{Conclusão}

Esse estudo contribuiu para incentivar a reflexão acerca dos sentimentos vivenciados pelos profissionais diante da Covid-19. Essa pandemia está disseminando problemas de saúde adicionais, como estresse, ansiedade, preocupação e medo em todo o mundo. As preocupações e medos influenciam os comportamentos diários, o que pode enfraquecer as estratégias de controle do Covid-19 e levar a mais morbidade e necessidades de saúde mental em nível global.

Nesse sentido, o atual cenário, voltados para a saúde mental desses trabalhadores demanda ainda mais atenção do poder público. Portanto, são necessários esforços imediatos, para minimizar os resultados negativos na saúde mental da população e entre os profissionais de saúde.

Logo, investir na assistência à saúde e, especialmente, na ciência em geral, é importante para que esse período seja 
breve e para que os profissionais possam estar preparados para enfrentamento dessa doença.

\section{Referências}

Adams, J. G., \& Ron, M. W. (2020). Supporting the Health Care Workforce During the COVID-19 Global Epidemic. Jama Network, 323 (15), 1439-1440. https://jamanetwork.com/journals/jama/fullarticle/2763136

Agência Nacional de Vigilância Sanitária. (2020). Covid 19: informações importantes. Anvisa. http://portal.anvisa.gov.br/documents/219201/4340 788/Perguntas+e+respostas+-+testes+para+Covid-19.pdf/9fe182c3-859b-475f-ac9f-7d2a758e48e7

Baker, M. G. et al. (2020). Estimating the burden of United States workers exposed to infection or disease: a key factor in containing risk of COVID-19 infection. Plos One. https://journals.plos.org/plosone/article?id=10.1371/journal.pone.0232452

Bardin, L. (2011). Análise de conteúdo. Edições 70.

Brasil. (2016). Resolução no 510, de 7 de abril de 2016. http://bvsms.saude.gov.br/bvs/saudelegis/cns/2016/res051 0_07_04_2016.html\#: :text=1\%20o\%20 Esta\%20Resolu\%C3\%A7\%C3\%A3o\%20disp\%C3\%B5e,existentes\%20na\%20vida\%20cotidiana\%2C\%20na

Brasil. (2020). Resolução da CNE/CES no 3, de 07 de novembro de 2001. Institui Diretrizes Curriculares Nacionais do Curso de Graduação em Enfermagem. http://portal.mec.gov.br/cne/arquivos/pdf/CES03.pdf

Brooks, S. K., et al. (2020). The psychological impact of quarantine and how to reduce it: rapid review of the evidence. The Lancet, 395 (10227), 912-920. https://www.thelancet.com/article/S0140-6736(20)30460-8/fulltext

Choi, H. C. (2019). The Refresher Training Curriculums Essential Subjects of Psychiatric Rehabilitation Facilities Mental Health Professionals Survey: focusing on mental health social worker. Journal of the Korean Society for Wellness, 14, (1), 219-229. https://www.researchgate.net/journal/19754051_Journal_of_the_Korean_society_for_Wellness

Costa, E. S., \& Moura, A. C. (2018). Motivação como fator de sucesso para a gestão de carreira: Revista de Carreiras e Pessoas, 8 (2), $212-226$.

Fontanella, B.J.B, et al. (2008). Amostragem por saturação em pesquisas qualitativas em saúde: contribuições teóricas. Cad de Saud Pub, 24 (1). $17-27$.

Harapan, et al (2020). Coronavirus Disease 2019 (COVID-19): a literature review. Journal of Infection and Public Health, 13 (5), 667-673. https://www.sciencedirect.com/science/article/pii/S1876034120304329

Ho, C., et al (2020). Mental health strategies to combat the psychological impact of COVID-19 beyond paranoia and panic. Annals Academy Medical of Singapure, 49 (3), 1-3. https://pesquisa.bvsalud.org/portal/resource/pt/mdl-32200399

Huang L, et al. (2020). Special attention to nurses’ protection during the COVID-19 epidemic. Crit Care, 24, (1), s13054-020-2841-7.

Maben, J., \& Bridges J. (2020). Covid-19: Supporting nurses' psychological and mental health. Journal of Clinical Nursing, 29 (15), 2742-2750.

Marchant, P., \& Ratinald, P. (2012). L'analyse de similitude apliqueéaux corpus textueles: les primairessocialistespourl' election présidentiellefrançaise. In: Actes des 11eme Journées internationales d'Analyse statistique des Données Textuelles, 13 (1), p.687-699. http://www.jadt2012.ulg.ac.be/

Mendes, A. M. (2007). Psicodinâmica do trabalho: teoria, método e pesquisas. Casa do Psicólogo; 2007.

Ministério da Saúde. (2020), Guia de Vigilância Epidemiológica: Vigilância de Síndromes Respiratórias Agudas COVID-19. Secretaria de Vigilância em Saúde. http://www.dive.sc.gov.br/notas-tecnicas/docs/af_gvs_coronavirus_6ago20_ajustes-finais-2.pdf

Neves, N. N. S., et al. (2020). O impacto da pandemia de COVID-19 nas emoç̃es dos trabalhadores da saúde e na aprendizagem professional. Research, Society and Development, 9,(12), e32391211367. Doi 10.33448/rsd-v9i12.11367

Pan, Y., et al. (2020). Serological immunochromatographic approach in diagnosis with SARS-CoV-2 infected COVID-19 patients. Journal of Infection, 81(1), 28-32. https://www.journalofinfection.com/article/S0163-4453(20)30175-4/fulltext

Saúde Mental e Atenção Psicossocial na Pandemia Covid-19 - Recomendações para gestores [Internet]. Fiocruz. https://portal.fiocruz.br/documento/saudemental-e-atencao-psicossocial-na-pandemia-covid-19-recomendacoes-para-gestores

Shanafelt, T., et al (2020) Understanding and Addressing Sources of Anxiety Among Health Care Professionals During the COVID-19 Pandemic. Jama Network, 323 (21), 2133-2134.

Turale, S et al. (2020). Challenging times: ethics, nursing and the COVID-19 pandemic. International Nursing Review, 67, (2), 164-16. https://onlinelibrary.wiley.com/doi/full/10.1111/inr.12598

World Health Organization (2011). Psychological first aid: Guide for field workers. Geneva: OMS

Yan Y et al. (2020). The First 75 Days of Novel Coronavirus (SARS-CoV-2) Outbreak: recent advances, prevention, and treatment. International Journal of Environmental Research and Public Health, https://www.ncbi.nlm.nih.gov/pmc/articles/PMC7177691

Yao, $\mathrm{H}$ et al. (2020). Patients with mental health disorders in the COVID-19 epidemic. The Lancet Psychiatry. 7 (4), 21. https://www.thelancet.com/journals/lanpsy/article/PIIS2215-0366(20)30090-0/fulltext.

Yeh-Li H \& Morais AM. (2020). COVID-19: o que aprendemos? Jornal Brai de Pneumolo ,46 (3), 202-216. https://www.scielo.br/pdf/jbpneu/v46n3/pt_18063713-jbpneu-46-03-e20200216.pdf. 